7. Durodoye OM, Mendlovic DB, Brenner RS, Morrow JS. Endocrine disturbances in empty sella syndrome: case reports and review of literature. Endocr Pract. 2005; 11: 120-4.

8. Rennenberg RJ, Bravenboer B, Wolffenbuttel BH. Empty sella syndrome as the cause of panhypopituitarism. Ned Tijdschr Geneeskd 2004; 148: $33-6$

9. Sartre J, Herranz de la Morena A, López Guzmán A, Gómez Pan A, Pallardo Sánchez LF. Silla turca vacía primaria: evaluación clínica, radiológica y hormonal. Rev Clin Esp 1992; 191: 481-4.

10. Donadio F, Barbieri A, Angioni R, Mantovani G, Beck-Peecoz P, Spada A, Lania AG. Patients with macroprolactinaemia: clinical and radiological features. Eur J Clin Invest. 2007; 37: 552-7.

\section{Leiomiosarcoma primario pulmonar de progresión lenta}

\section{Sr. Director:}

El leiomiosarcoma es un tumor infrecuente que procede de células musculares lisas, afecta en muy raras ocasiones al pulmón de forma primaria, siendo más frecuente la localización en el tracto gastrointestinal o genital femenino; las lesiones pulmonares suelen ser metástasis de dichas localizaciones. El diagnóstico se apoya fundamentalmente en la clínica, siendo necesario la realización de pruebas de imagen y estudio anatomopatológico para confirmarlo. Su presentación como una masa pulmonar en los estudios de imagen, a menudo supone un reto diagnóstico. A continuación presentamos el caso de una paciente diagnosticada de leiomiosarcoma primario pulmonar a partir del hallazgo casual como masas de localización intraparenquimatosa, en la radiografía simple de tórax.

Mujer de 68 años de edad, que acudió al servicio de Urgencias de nuestro hospital por haber presentado un esputo hemoptoico autolimitado.

Tenía antecedentes personales de crisis renoureterales de repetición y diabetes mellitus tipo 2. Ocho años antes fue estudiada por lesiones pulmonares. La paciente refería contacto con perros en la infancia y como antecedentes familiares, madre intervenida en dos ocasiones de quistes hidatídicos.

A su llegada al hospital se encontraba asintomática y refería un episodio de hemoptisis de escasa cuantía, que no se acompañó de dolor torácico, disnea, ni fiebre. Describía así mismo, un único episodio de similares características en los meses previos al que no dio importancia.

La paciente se encontraba eupneica, con saturaciones de oxigeno basales del $98 \%$, afebril, con buena coloración de piel y mucosas, y sin hallazgos patológicos en la auscultación pulmonar. No hubo otras alteraciones de interés en la exploración física.

En el servicio de Urgencias se realizó una analítica básica con hemograma, bioquímica y coagulación que fueron normales. La radiografía postero-anterior de tórax mostraba una imagen de aumento de densidad en región basal derecha, de bordes nítidos, en contacto con la pared torácica, y otra imagen de similares características en pulmón izquierdo (Fig. 1). Ante estos hallazgos se decidió el ingreso de la paciente en el Servicio de Medicina Interna para completar estudio, realizándose durante el mismo una TC torácica (Fig. 2).

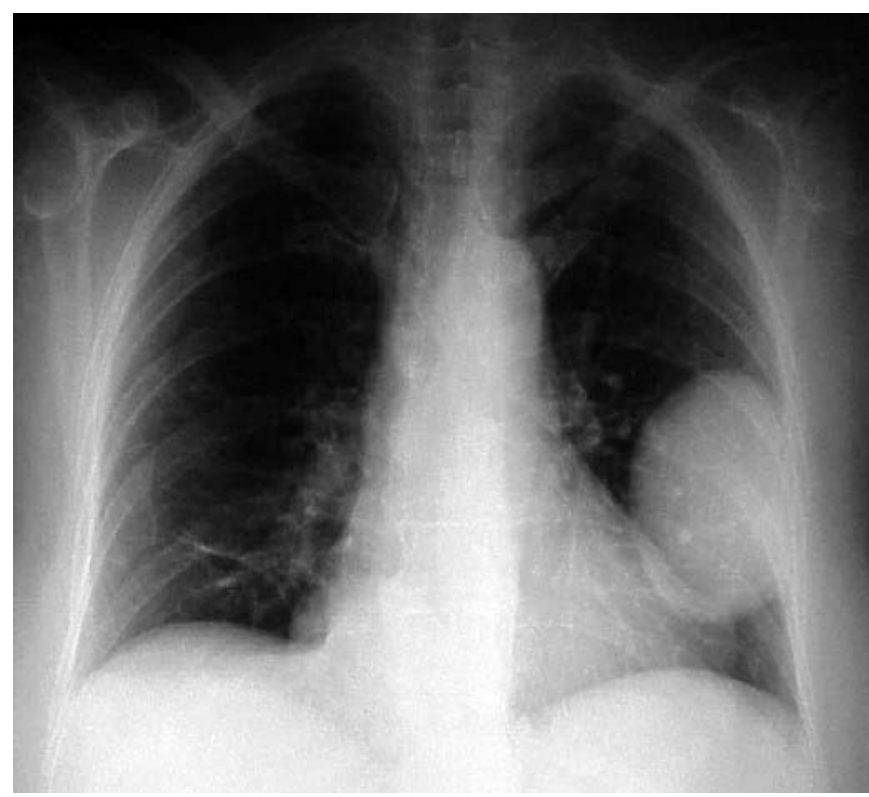

Fig. 1.

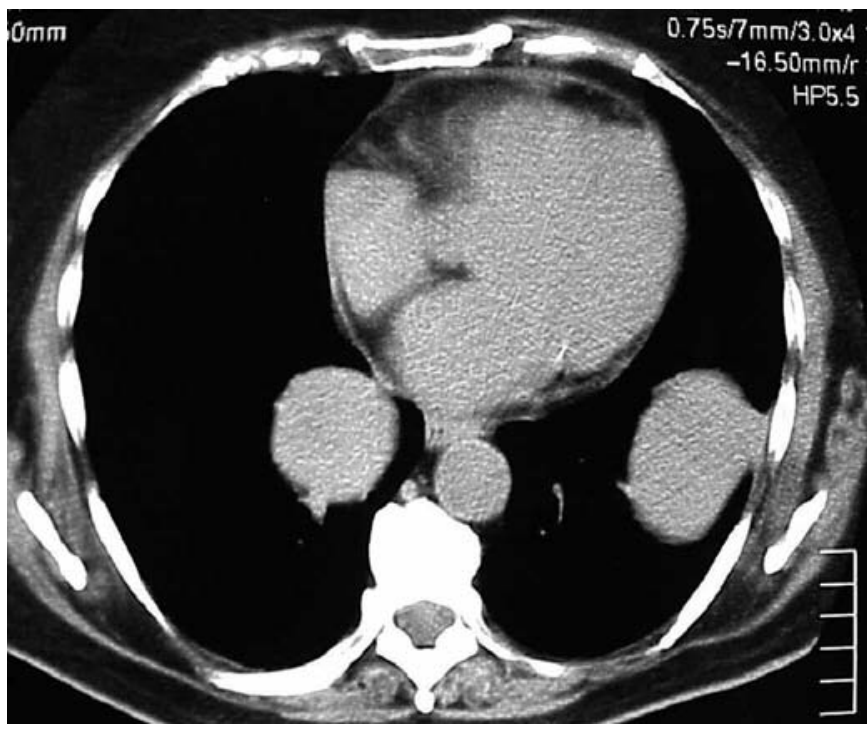

Fig. 2.

En la TC tóraco-abdominal, realizada durante su ingreso, se visualizó una tumoración basal derecha que ocupaba el receso pleuro-acigo-esofágico $(5,5 \mathrm{~cm})$ y otra tumoración de similares características en hemitórax izquierdo $(7 \mathrm{~cm})$. Ambas lesiones en extenso contacto con la superficie pleural (Fig. 2). A nivel abdominal no se encontraron alteraciones. Se realizó PAAF guiada por TC de una de las masas, la cual fue informada como tumor mesenquimal benigno de células fusiformes. El resto de las pruebas diagnósticas realizadas (analítica completa con marcadores tumorales y serología de hidatidosis), resultaron normales.

Al revisar la historia clínica de la paciente, se constató que fue estudiada 8 años atrás por lesiones pulmonares, realizándose una TC tóraco-abdominal donde se objetivaron masas pulmonares similares, aunque de menor tamaño a las referidas en la TC actual. En aquél momento se realizó PAAF que fue sugerente de 
proceso inflamatorio.

De acuerdo con el Servicio de Cirugía Torácica, se decidió intervenir a la paciente. Se practicó una lobectomía superior izquierda postergándose la extirpación de la tumoración basal derecha. El resultado anatomo-patológico fue compatible con leiomiosarcoma pulmonar de bajo grado de malignidad.

El leiomiosarcoma primario es una neoplasia infrecuente (1$4 \%$ de los sarcomas primarios de tejidos blandos) (1). Es un tumor maligno de células musculares lisas, que afecta más veces al tracto gastrointestinal y genital femenino (2). En el pulmón, puede ser de origen endobronquial, vascular y parenquimatoso y tener localización periférica o central. Los de origen parenquimatoso son los más frecuentes y forman masas bien delimitadas que pueden alcanzar grandes dimensiones $(3,4)$. Muchos de estos tumores cursan de forma asintomática, constituyendo un hallazgo incidental, también pueden presentarse con tos, dolor torácico, o hemoptisis (3).

Es infrecuente que metastaticen por vía linfática, por lo que las lesiones pulmonares no suelen acompañarse de adenopatías hiliares o mediastínicas, como en el carcinoma. El pulmón es también uno de los asientos más frecuentes de metástasis de todos los sarcomas de tejidos blandos, por lo que siempre debemos excluir esta posibilidad y confirmar el origen primario del tumor (5).

El diagnóstico se basa en técnicas inmunohistoquímicas e histológicas donde se observan células fusiformes con proliferación en láminas y núcleos pleomórficos (1-4,6).

La resección completa del tumor disminuye la aparición de recidiva local (1) y es considerada como uno de los factores mas importantes para el pronóstico y para la predicción de recidivas, otros factores a considerar son el grado histológico y el estadio tumoral $(6,7)$. Este tipo de tumores son débilmente sensibles a la radioterapia, y responden pobremente a la quimioterapia (8).

\section{P. Benito García, A. Fuertes Martín, E. Santos Corraliza, S. Bahamonde Cabria, R. Merchán Rodríguez}

Servicio de Medicina Interna II. Hospital Clínico Universitario. Salamanca

1. Matsumoto J, Sugiuria H, Morikawa T, Kaji M, Kondo S, Okushiba S, et al. A case of Primary Leiomiosarcoma of the Chest Wall Successfully Resected under the Video-assisted Thoracoscopic Approach. Ann Thorac Cardiovasc Surg 2001; 7: 368-70.

2. Pérez Villa J, Muntané MJ, Venegas MP, Viladot J. Leiomiosarcoma de laringe: a propósito de un nuevo caso. Acta Otorrinolaringol Esp 2001; 52: 254-7.

3. Fraser RS, Colman N, Müller NL, Pare PD. Neoplasias pulmonares. En Fundamentos de las enfermedades del tórax. Ed. Masson $3^{\mathrm{a}}$ ed. Barcelona; 2006. p. 393-4.

4. Grande Sáez C, González Quijada S, Dueñas Gutiérrez C, Marrero Calvo M, Martín Ezquerro, Ceballos Barón I. Leiomosarcoma multicéntrico en paciente VIH. An Med Interna 2002; 19: 377-378.

5. Petrov DB, Vlassov VI, Kalaydjiev GT, Plochev MA, Obretenov ED, Stanoev VI, et al. Primary pulmonary sarcomas and carcinosarcomas posoperative results and comparative survival analysis. Eur J Cardiothorac Surg 2003; 23: 461-6.

6. Redón Henao J, Sánchez Mora N, Álvarez Fernández E. Leiomiosarcoma de mediastino anterior. Rev Esp Patol 2004; 37: 99-103.

7. Regnard JF, Icard P, Guibert L, Montpreville VT, Magdeleinat P, Levasseur P. Prognostic factors and results after surgical treatment of primary sarcomas of the lung. The Annals of Thoracic Surgery 1999; 68: 227-31.

8. Buch Villa E, Arnau A, Garcia Aguado R, Sabater V, Canto A, Fernandez Centeno A, y cols. Leiomiosarcoma pulmonar. Arch Bronconeumol 1994; 30: 465-7.
Asociación entre el uso de fármacos antipsicóticos y las alteraciones metabólicas en población adulta española

\section{Sr. Director:}

Es conocido que las alteraciones metabólicas como la obesidad, diabetes o dislipemia aumentan el riesgo de padecer eventos cardiovasculares (1-2). En España, los datos aportados por la Sociedad Española para el Estudio de la Obesidad (SEEDO) indican que la prevalencia poblacional de la obesidad es superior al $14 \%$, con una clara tendencia al incremento progresivo alcanzando su máximo entre los 40 y los 50 años (3-4). En otro aspecto, la utilización de fármacos antipsicóticos (ATP) está aumentando, y produce efectos secundarios en el metabolismo de la glucosa y de los lípidos, ocasionando un aumento del peso (5-6). No obstante, existen sensibles controversias entre los diferentes autores, puesto que los efectos de clozapina, olanzapina y risperidona pueden producir un aumento de los niveles de colesterol y triglicéridos (6), o bien, un mayor riesgo de padecer diabetes mellitus (2). La influencia de cada medicamento, resulta difícil de cuantificar, como también lo es, discernir si determinadas alteraciones biológicas son causa o efecto del exceso de peso (5). El objeto del estudio fue evaluar la asociación entre las principales alteraciones metabólicas (diabetes, dislipemia en presencia de obesidad) y el uso regular de fármacos ATP típicos y atípicos, en pacientes adultos atendidos por cinco equipos de atención primaria en un ámbito poblacional español.

Se realizó un estudio de carácter retrospectivo y multicéntrico, con muestreo sistemático, a partir de los registros médicos de pacientes adultos seguidos en régimen ambulatorio y en situación de práctica clínica habitual. La población de estudio estuvo formada por cinco equipos de atención primaria gestionados por Badalona Serveis Assistencials SA. El tipo de organización de los equipos es de carácter reformado, con gestión de titularidad pública y provisión de servicios privada, concertados con el Servicio Catalán de la Salud (CatSalut). Se incluyeron en el estudio todos los pacientes atendidos entre el 1 de julio de 2004 y el 30 de junio de 2005, con edad superior a 20 años. Se consideraron pacientes en uso regular de ATP los que: a) iniciaron el tratamiento 3 meses antes del estudio, b) estaban en el programa de prescripciones crónicas del centro, c) acudieron con regularidad a las citas programadas para la obtención de las recetas médicas, y d) presencia de un registro constatado de la dosis diaria, el intervalo de tiempo y la duración de cada tratamiento administrado. Fueron excluidos los pacientes que no acudieron a los centros, los desplazados o fuera de zona y los que sufrían enfermedades mentales incapacitantes. Se consideró obesidad, por la presencia de un exceso de peso por acumulación de tejido graso, cuando el índice de masa corporal (IMC, $\mathrm{kg} / \mathrm{m}^{2}$ ) fue superior a 29 (criterios de la NCEP8). Además, se obtuvo información de la prescripción farmacológica de medicamentos ATP (atípicos y típicos), emitida en las historias clínicas informatizadas según las recomendaciones realizadas por el facultativo o especialista de referencia. Los diferentes principios activos se agruparon en cinco categorías: a) atípicos (amisulprida, clozapina, quetiapina, ziprasidona); b) olanzapina; c) risperidona; d) típicos (clorpromazina, clotiapina, flufenazina, levomepromazina, perfenazina, periciazina, pimozida, pipotiazina, tiaprida, tioproperazina, tioridazina, zuclopentixol); y e) haloperidol. Las diferentes comorbilidades, diagnósticos o motivos de consulta en población historiada fueron cuantificados a partir de la Clasificación Internacional de la Atención Primaria (CIAP: Lamberts y Wood), ver tabla 1. Los datos fueron validados, restándose la confidencialidad de los registros marcada por la ley. Se realizó un análisis estadístico descriptivo, con valores de media, desviación típica (DE), intervalos de confianza del 95\% 